\title{
Communication
}

\section{Dobutamine Stress Echocardiography in Chronic Kidney Disease Patients Candidates for Renal Transplantation}

\author{
Abdel Mohsen Mostafa Aboualia \\ Cardiology Department, Faculty of Medicine, Al-Azhar University, Cairo, Egypt \\ Email address: \\ Abdelmohsen1@hotmail.com
}

To cite this article:

Abdel Mohsen Mostafa Aboualia. Dobutamine Stress Echocardiography in Chronic Kidney Disease Patients Candidates for Renal Transplantation. Cardiology and Cardiovascular Research. Vol. 4, No. 3, 2020, pp. 164-168. doi: 10.11648/j.ccr.20200403.22

Received: September 5, 2020; Accepted: September 17, 2020; Published: September 23, 2020

\begin{abstract}
Background: Dobutamine stress echocardiography (DSE) is used for induction of ischemia in different population. One of those is chronic kidney disease patients before renal transplantation for pre-operative risk assessment. Aim of the work: to evaluate the difference in dobutamine stress echocardiography between those with chronic kidney disease (CKD) on regular dialysis and patient with no known history of chronic renal impairment. Methods: From February 2016 to January 2017 , twenty consecutive chronic kidney disease patients prepared for renal transplantation without history suggestive of ischemic heart disease and 40 consecutive patients with without history of renal impairment. Their heart rate, dobutamine dose, atropine dose and adverse side effects were compared between the two groups. Results: Both groups were matched in age and risk factors. Peak heart rate $(\mathrm{HR})(133.7 \pm 16.99$ vs. $144.08 \pm 10.99 \mathrm{~b} / \mathrm{m}, P=0.012)$, time to target heart rate was $(17.75 \pm 3.29 \mathrm{vs} .13 .05 \pm 2.95 \mathrm{~min}$, $\mathrm{p}=0.001)$ and dobutamine and atropine doses were higher in chronic kidney disease group than other group. Adverse reactions present in both groups but more incidence in CKD group. Conclusion: Chronic kidney disease patients response to dobutamine when underwent dobutamine stress echocardiography showed delayed effect of it with more dobutamine and atropine doses needed and more adverse reactions including Bezold-Jarisch reflex.
\end{abstract}

Keywords: Dobutamine Stress Echocardiography, Chronic Kidney Disease, Bezold-Jarisch Reflex

\section{Introduction}

Chronic kidney disease (CKD) is a worldwide disease affecting more than 20 million adults and with increasing prevalence of obesity, old age and diabetes, this number is expected to be the double by 2030 [1]. Cardiovascular disease is the leading cause of mortality and morbidity in patients with CKD [2]. The cardiovascular mortality is $15-30$ times higher than the general population [3]. The prevalence of coronary artery disease (CAD) is high in CKD although less predicted conventional risk factors and low sensitivity of chest pain (65\%) [4]. So, stress testing is used for risk stratification in CKD patients prepared for renal transplantation pre-operatively.

There is no agreement in guidelines about the optimal approach of cardiovascular evaluation in renal transplantation candidates. American College of Cardiology (ACC) and American Heart Association (AHA) guidelines on perioperative cardiovascular evaluation in population undergoing non-cardiac surgery published in 2014 in asymptomatic patients with a functional capacity $\geq 4$ metabolic equivalents) that no need for further tests. While, noninvasive tests performed for patients at elevated risk regardless of functional status based on the 2012 AHA/ACC scientific statement regarding cardiac evaluation in renal transplantation candidates [5-6]. These risk factors include diabetes mellitus, previous cardiovascular disease, $>1$ year on dialysis, left ventricular hypertrophy (LVH), age $>60$ years, smoking, hypertension, and dyslipidemia with presence of $\geq 3$ risk factors is reasonable threshold for noninvasive testing [6]. The recommendation of American Society of Transplantation is that patients with diabetes, known to be ischemic heart disease or had an abnormal electrocardiogram (ECG), or their age $\geq 50$ years should be classified as high-risk for $\mathrm{CAD}$ and should be referred for a cardiac stress test, if positive result referred for coronary angiography [7]. 
Dobutamine Stress echocardiography (DSE) is a procedure that evaluate myocardial ischemia in a manner simulating the exercise by increased cardiac output, heart rate and myocardial oxygen demand.

\section{Patients and Methods}

\subsection{Study Design}

In this observational case-control study twenty consecutive patients with chronic kidney disease planned for renal transplantation referred for a pre-operative DSE from February 2016 to January 2017 at Naser Instiute for Research and Treatment. Another 40 consecutive patients without history of kidney disease who also refereed for DSE. Clinical indications for DSE were the evaluation of patients with chest pain and intermediate pretest probability for CAD. Exclusion criteria were known to be ischemic patients (a recent myocardial infarction, unstable angina), uncontrolled arrhythmia (ventricular tachycardia or atrial fibrillation with the rapid ventricular response) and significant aortic stenosis. No medications were stopped before the procedure. The study was approved by the local ethical committee and an informed consents were obtained from all patients.

\subsection{Dobutamine Stress Echocardiographic Procedure}

Baseline transthoracic echocardiography was done using GE Vivid S6 Ultrasound System with M4S phased array probe (GE Healthcare, Wauwatosa, WI, USA) was performed in all the standard views (apical 4 chamber, apical 2 chamber, parasternal long axis and parasternal short axis views) ECG guided were done and acquired on the vendor archive [8].

\subsection{Standard Dobutamine-Atropine Infusion Protocol}

After IV line was inserted, dobutamine was infused with 3-5 minutes dose increments, starting from $5 \mu \mathrm{g} / \mathrm{kg} / \mathrm{min}$ and increasing to $10,20,30$ and $40 \mu \mathrm{g} / \mathrm{kg} / \mathrm{min}$, then recovery stage with acquiring video loops at baseline, low-dose (20 $\mu \mathrm{g} / \mathrm{kg} / \mathrm{min}$ ), peak when reach target heart rate either with dobutamine alone or with adding atropine. Throughout and after dobutamine infusion the ECG was monitored. Target heart rate (THR) was calculated from the following equation $\mathrm{THR}=(220$-age $) * 85$. When THR not reached, atropine was given to the continuing $40 \mu \mathrm{g} / \mathrm{kg} / \mathrm{min}$ dobutamine infusion stage if no contraindications such as glaucoma or prostatic enlargement were present. Atropine was given at dose $0.5 \mathrm{mg}$ bolus then $10 \mathrm{ml}$ flush of saline and repeated at dose $0.25 \mathrm{mg}$ for maximum dose $2 \mathrm{mg}$ [9]. The infusion was terminated after reaching the maximal dose or at any of the following points:

1) More than $85 \%$ of the age-predicted maximal HR

2) Marked elevation of hypertension (SBP of $>240 \mathrm{~mm} \mathrm{Hg}$ or diastolic BP of $>120 \mathrm{~mm} \mathrm{Hg}$ )

3) Significant cardiac arrhythmias (sustained ventricular tachycardia)

4) Severe typical chest pain

5) Horizontal or downsloping ECG ST depression of $\geq 1$ $\mathrm{mm}$ ( $80 \mathrm{~ms}$ after the J point) or ST-segment elevation (in the absence of $\mathrm{Q}$ wave) more than or equal $\mathrm{mm}$

6) New regional wall motion abnormalities in more than two adjacent segments

7) On patient request due to intolerable symptoms [10].

\subsection{Images Interpretation}

After the study end off-line assessment of stored digitized echocardiographic loops and displayed in a standard quad-screen digital format that for easy side by side comparison for each patient by the operator. Regional wall motion was assessed according to the recommendations of the American Society of Echocardiography using a 17-segment model and wall motion was graded as follows: $1=$ normal, $2=$ hypokinetic, $3=$ akinetic, and 4=dyskinetic [8]. Wall motion score index were calculated by dividing the sum of wall motion scores by the number of examined segments at baseline and at peak of stress test. The test was considered positive when wall motion in any segment deteriorated $>1$ grade, as all patients showed baseline normokinetic segments [11]. Studies were identified as non-conclusive if the patient cannot reach $85 \%$ age-predicted maximal HR in the absence of inducible ischemia. The baseline heart rate and maximal reached HR was recorded. Offline measurement of EF using biplane modified Simpson's methods for baseline and peak loops were calculated, the time till reach of peak HR was measured using the stop watch on the vendor. The adverse symptoms and arrhythmia was recorded in special sheets. Bezold-Jarisch reflex was considered present when the heart rate decreases with continuous incremental dobutamine infusion. It was transient with spontaneously relieved or 0.5 $\mathrm{mg}$ atropine bolus IV if persists more than 1 minute [12].

\subsection{Statistical Analysis}

Data management and statistics were done using IBM statistical package for social sciences, version 22.0 (IBM corporation and others, USA). Numerical data were expressed as mean \pm standard deviation (SD). Categorical data were expressed as number and percentage. Independent $t$ test was used when comparing between two means. Chi-square (x2) test of significance was used in order to compare proportions between categorical parameters. The p-value was considered significant if $\mathrm{P}$-value $<0.05$ and highly significant if $\mathrm{P}$-value $<0.001$.

\section{Results}

The patients included in this study were 60 patients with male predominance (49 patient, 80.7\%) with their age $54.45 \pm 8.88$ years. Diabetes mellitus was present in 5 patients (8.3\%), hypertensions in 24 patients (40\%) and smoking in 26 patients $(43.3 \%)$ while chronic kidney disease was present in one third of cases (20 patients). The average baseline heart rate was $66.73 \pm 12.05 \mathrm{~b} / \mathrm{m}$ while peak HR was $140.62 \pm 14.04$. the maximum reached dose of dobutamine was $37.17 \pm 5.24$ $\mu / \mathrm{kg} / \mathrm{min}$ while the maximum atropine dose was $0.54 \pm 0.42$ 
mg. the patients needed $14.62 \pm 3.77$ minute to reach the target heart rate. The calculated EF was $58.25 \pm 3.38 \%$ at baseline and $69.35 \pm 5.19 \%$ at peak of the test. The resting WMSI was 1 while the peak WMSI was $1.10 \pm 0.12$. The adverse reactions of dobutamine were reported in table 2 .

Table 1. Demographic characters and risk factors of the studied population.

\begin{tabular}{ll}
\hline Parameter & mean \pm SD/frequency $(\%)$ \\
\hline Age (years) & $54.45 \pm 8.88$ \\
Sex (F) & $11(18.3 \%)$ \\
Diabetes mellitus F (\%) & $5(8.3 \%)$ \\
Hypertension F (\%) & $24(40 \%)$ \\
Smoking F (\%) & $26(43.3 \%)$ \\
CKD F (\%) & $20(33.3 \%)$ \\
\hline
\end{tabular}

Table 2. Baseline and peak parameters.

\begin{tabular}{ll}
\hline Parameter & mean \pm SD/frequency (\%) \\
\hline Resting HR $(\mathrm{b} / \mathrm{m})$ & $66.73 \pm 12.05$ \\
Peak HR $(\mathrm{b} / \mathrm{m})$ & $140.62 \pm 14.04$ \\
Dobutamine rate $(\mu / \mathrm{kg} / \mathrm{min})$ & $37.17 \pm 5.24$ \\
Atropine dose $(\mathrm{mg})$ & $0.54 \pm 0.42$ \\
Time to THR (minutes) & $14.62 \pm 3.77$ \\
Baseline EF (\%) & $58.25 \pm 3.38$ \\
Peak EF (\%) & $69.35 \pm 5.19$ \\
Resting WMSI & $1.0 \pm 0.0$ \\
Peak WMSI & $1.10 \pm 0.12$ \\
Nausea F (\%) & $49(81.7 \%)$ \\
Vomiting F (\%) & $20(33.3 \%)$ \\
Palpitation F (\%) & $31(51.7 \%)$ \\
Chest pain F (\%) & $28(46.7 \%)$ \\
PVCs F (\%) & $31(51.7 \%)$ \\
APCs F $(\%)$ & $40(66.6 \%)$ \\
Runs of V tach F (\%) & $3(5 \%)$ \\
Bezold-Jarisch reflex & $7(11.7 \%)$ \\
\hline
\end{tabular}

When comparing the patients with chronic kidney disease to those without chronic kidney disease the age, sex, diabetes mellitus, hypertension and smoking were matched between both groups (table 3 ).

Table 3. Comparison between demographic parameters and risk factors based on chronic kidney disease presence.

\begin{tabular}{llll}
\hline Parameters & No CKD $(\mathbf{n}=40)$ & CKD $(\mathbf{n = 2 0})$ & P value \\
\hline Age (years) & $54.48 \pm 8.57$ & $54.40 \pm 9.70$ & 0.47 \\
Sex (F) & $5(12.5 \%)$ & $6(30 \%)$ & 0.09 \\
Diabetes mellitus F (\%) & $3(7.25 \%)$ & $2(10 \%)$ & 0.7 \\
Hypertension F (\%) & $17(42.5 \%)$ & $7(35 \%)$ & 0.5 \\
Smoking F (\%) & $18(45 \%)$ & $8(40 \%)$ & 0.7 \\
\hline
\end{tabular}

The baseline HR was matched while the peak HR was reduced in CKD group which may be explained by higher mean age of them. The dobutamine doses needed to reach the target heart rate and needed atropine dose were higher in CKD group ( $40 \mathrm{vs} 35.75 \pm 5.94 \mu / \mathrm{kg} / \mathrm{min}$ and $0.91 \pm 0.1$ vs $0.36 \pm 0.38$ $\mathrm{mg}$ respectively). Also, the time needed to reach THR was higher in CKD (17.75 \pm 3.29 vs $13.05 \pm 2.95$ minutes) with all these difference is highly statically significant. No significant difference between EF nor WMSI either at baseline or at peak of test. The adverse symptoms was not significant between the two group except nausea which is significantly dominant in CKD. Ventricular ectopics were higher in CKD group with $p$ value 0.04 . Bezold-Jarisch reflex was highly significant present in CKD group (table 4).

Table 4. Comparison of baseline and peak parameters between the both groups.

\begin{tabular}{llll}
\hline Parameters & No CKD $(\mathbf{n}=\mathbf{4 0})$ & CKD $(\mathbf{n}=\mathbf{2 0})$ & P value \\
\hline Resting HR $(\mathrm{b} / \mathrm{m})$ & $65.08 \pm 12.90$ & $70.05 \pm 9.59$ & 0.51 \\
Peak HR $(\mathrm{b} / \mathrm{m})$ & $144.08 \pm 10.99$ & $133.70 \pm 16.99$ & 0.012 \\
Dobutamine rate $(\mu / \mathrm{kg} / \mathrm{min})$ & $35.75 \pm 5.94$ & $40.00 \pm 0$ & 0.001 \\
Atropine dose $(\mathrm{mg})$ & $0.36 \pm 0.38$ & $0.91 \pm 0.19$ & 0.001 \\
Time to THR (minutes) & $13.05 \pm 2.95$ & $17.75 \pm 3.29$ & 0.001 \\
Baseline EF (\%) & $57.40 \pm 2.43$ & $59.95 \pm 4.33$ & 0.003 \\
Peak EF (\%) & $70.10 \pm 4.61$ & $67.85 \pm 6.03$ & 0.123 \\
Resting WMSI & $1.00 \pm 0$ & $1.00 \pm 0$ & 1 \\
Peak WMSI & $1.09 \pm 0.11$ & $1.12 \pm 0.14$ & 0.405 \\
Nausea F (\%) & $29(72.5 \%)$ & $20(100 \%)$ & 0.009 \\
Vomiting F (\%) & $13(325 \%)$ & $7(35 \%)$ & 0.8 \\
Palpitation F (\%) & $20(50 \%)$ & $11(55 \%)$ & 0.7 \\
Chest pain F (\%) & $20(50 \%)$ & $8(40 \%)$ & 0.4 \\
PVCs F (\%) & $17(42.5 \%)$ & $14(70 \%)$ & 0.04 \\
APCs F (\%) & $25(62.5 \%)$ & $15(75 \%)$ & 0.06 \\
Runs of V tach F (\%) & $2(5 \%)$ & $1(5 \%)$ & 1 \\
Bezold-Jarisch reflex & $2(5 \%)$ & $5(25 \%)$ & 0.02 \\
\hline
\end{tabular}

\section{Discussion}

Because end stage renal (especially those on regular dialysis) associated with other comorbidities such as hypertension, diabetes mellitus, dyslipidemia, and tobacco so CAD is highly prevalent in those patients $(38 \%$, with a relative risk of 5 - to 20 - fold) [13]. There is cardiorenal axis as both atherosclerosis and end stage renal disease (ESRD) are inflammatory state beside uremia accelerate progression of CAD [14]. In CKD and ESRD activated renin-angiotensinaldosterone system leads to endothelial dysfunction and oxidative stress that predispose to plaque formation, fissuring and extensive heterotopic alcification [13]. In a meta analysis the accuracy of dobutamine stress echocardiogram and Single-photon emission computed tomography (SPECT) in detecting significant obstructive CAD ( $\geq 70 \%$ stenosis) in renal transplantation candidates is not statistically significant beside prognostic value of induced ischemia by dobutamine stress echocardiogram (DSE) and the local availability in many echocardiography machines, short half life of dobutamine (2 minutes) and available beta-blocker intravenous as antidote if arrhythmia is persistent [15].

This study while assessed and evaluated the difference between CKD and non-CKD when both exposed to DSE found that the peak HR was reduced in CKD group which may be explained by higher mean age of them and delayed time to reach the peak HR. The dobutamine doses needed to reach the target heart rate and needed atropine dose were higher in CKD group.

The previous study on DSE including the different meta-analysis [15-19] (studied the prognostic and diagnostic capabilities but not the dose of drugs nor the response to dobutamine.

The delayed response to dobutamine and need to use higher doses of atropine may be related to effect of metabolites in ESRD that may blunt the heart response to dobutamine as dobutamine is metabolized by the liver and no role of kidney 
in dobutamine metabolism. Although previous report in pediatrics of delayed clearance of dobutamine in infants with serum creatinine concentrations $>2 \mathrm{mg} / \mathrm{dl}$ [20]. This observation alerts the alternative protocol of accelerated DSE [21] where atropine is used at dobutamine dose of $20 \mu / \mathrm{kg} / \mathrm{min}$ that could decrease the procedure time and dose of dobutamine used.

In this study no difference in diagnostic capability of DSE between CKD and non-CKD patients as RWMSI in both groups were comparable.

CKD potentiates the arrhythmogenic effect of DSE in the form of ventricular ectopics and this could be explained by the electrolyte disturbance in CKD and the regulation of adrenergic receptors in CKD patients.

Bezold-Jarisch reflex (BJR) was significant present in CKD group and this reminds us of the potential complications and importance of closely monitoring patients during dobutamine infusion. Although Bezold-Jarisch reflex (BJR) is a benign condition that can be abolished by dobutamine infusion cessations or atropine injection, but there were reported severe cases of DSE induced asystole [12]. BJR has been described in patients with heart failure or inferior or posterior wall ischemia. BJR is a cardioprotective reflex through decreasing afterload and mechanical work resulting in reduction of myocardial oxygen demand. In addition, the patients developed BJR were diabetic (all of non-CKD and 2 of 5 in CKD) with predisposition to autonomic dysfunction and dominant vagal tone. But, coronary angiography in those patients may add a value.

\section{Limitations}

The current study limitations are: small sample size and the single-center experience.

\section{Conclusion}

Chronic kidney disease patients response to dobutamine differ than non-CKD patients high doses of dobutamine needed and mandatory need to use atropine. Accelerated DSE is an alternative protocol to be used instead of ordinary DSE. Exaggerated response to dobutamine is present with close monitoring is needed.

\section{References}

[1] U.S. Renal Data System. USRDS 2011 annual data report: Atlas of chronic kidney disease and end-stage renal disease in the United States. 2011.

[2] Bangalore S: Stress testing in patients with chronic kidney disease: The need for ancillary markers for effective risk stratification and prognosis. Journal of Nuclear Cardiology. $2016 ; 23 ; 570-574$

[3] Go AS, Chertow GM, Fan D, et al.: Chronic kidney disease and the risks of death, cardiovascular events and hospitalization. N Engl J Med 2004; 351: 1296-305.
[4] Schmidt A, Stefenelli T, Schuster E, et al.: Informational contribution of noninvasive screening tests for coronary artery disease in patients on chronic renal replacement therapy. Am J Kidney Dis. 2001; 37: 56-63.

[5] Fleisher LA, Fleischmann KE, Auerbach AD, et al.: 2014 ACC/AHA guideline on perioperative cardiovascular evaluation and management of patients undergoing noncardiac surgery: a report of the American College of Cardiology/American Heart Association Task Force on Practice Guidelines. J Am Coll Cardiol. 2014; 64: e77e137.

[6] Lentine KL, Costa SP, Weir MR, et al.: Cardiac disease evaluation and management among kidney and liver transplantation candidates: a scientific statement from the American Heart Association and the American College of Cardiology Foundation. J Am Coll Cardiol. 2012; 60: 434480 .

[7] Karthikeyan V, Ananthasubramaniam K: Coronary risk assessment and management options in chronic kidney disease patients prior to kidney transplantation. Curr Cardiol Rev. 2009; 5 (3): 177-186. doi: 10.2174/157340309788970342.

[8] Lang RM, Badano LP, Mor-Avi V, et al.: Recommendations for cardiac chamber quantification by echocardiography in adults: an update from the American Society of echocardiography and the European Association of Cardiovascular imaging. J Am Soc Echocardiogr. 2015; 28: 1-39 e14.

[9] Pellikka P, Nagueh S, Elhendy A, et al.: American Society of Echocardiography recommendations for performance, interpretation, and application of stress echocardiography. J Am Soc Echocardiogr. 2007; 20: 1021-1041.

[10] Lim YC, Teo SG, Poh KK: ST-segment changes with exercise stress. Singapore Med J. 2016; 57 (7): 347-353. doi: 10.11622/smedj.2016116.

[11] Senior R, Lahiri A: Enhanced detection of myocardial ischemia by stress dobutamine echocardiography utilizing the "biphasic" response of wall thickening during low and high dose dobutamine infusion. J Am Coll Cardiol 1995; 26: 26-32.

[12] Parent ME, Lepage S: A Heart Stopping Case of the Bezold-Jarisch Reflex. Case Rep Cardiol. 2015; 2015: 359401. doi: 10.1155/2015/359401.

[13] Bhatti NK, Karimi GK, Paz Y, et al.: Diagnosis and Management of Cardiovascular Disease in Advanced and End-Stage Renal Disease. J Am Heart Assoc. 2016; 5 (8): e003648. doi: 10.1161/JAHA.116.003648.

[14] Shirani J, Meera S, Dilsizian V: The Cardiorenal Axis: Myocardial Perfusion, Metabolism, and Innervation. Current Cardiology Reports. 2019; 21: 60.

[15] Wang LW, Fahim MA, Hayen A, et al.: Cardiac testing for coronary artery disease in potential kidney transplant recipients a systematic review of test accuracy studies. Am J Kidney Dis. 2011; 57: 476-487.

[16] Sharma R, Pellerin D, Gaze DC, et al.: Dobutamine stress echocardiography and the resting but not exercise electrocardiograph predict severe coronary artery disease in renal transplant candidates. Nephrology Dialysis $\begin{array}{llll}\text { Transplantation. } 2005 ; & 20 & \text { (10): } & \text { 2207-2214. }\end{array}$ https://doi.org/10.1093/ndt/gfi005. 
[17] Rakhit DJ, Armstrong KA, MAppStat EB, et al.: Risk stratification of patients with chronic kidney disease: Results of screening strategies incorporating clinical risk scoring and dobutamine stress echocardiography American Heart Journal. 2006: 152 (2): 363-370.

[18] Bergeron S, Hillis GS, Haugen EN, et al.: Prognostic value of dobutamine stress echocardiography in patients with chronic kidney disease. Am Heart J. 2007; 153: 385-391.

[19] Ramphul R, Fernandez M, Firoozi S, et al.: Assessing cardiovascular risk in chronic kidney disease patients prior to kidney transplantation: clinical usefulness of a standardised cardiovascular assessment protocol. BMC Nephrology. 2018: 19: 2.

[20] Pacifici GM: Clinical pharmacology of dobutamine and dopamine in preterm neonates. MedicalExpress (São Paulo, online) $\quad$ [Internet]. 2014; $1 \quad$ (5): 275-283. https://doi.org/10.5935/MedicalExpress.2014.05.12.

[21] San Román JA, Sanz-Ruiz R, Ortega JR, et al. Safety and predictors of complications with a new accelerated dobutamine stress echocardiography protocol. J Am Soc Echocardiogr 2008; 21: 53-57. 